\title{
DENSE EMBEDDINGS OF SIGMA-COMPACT, NOWHERE LOCALLY COMPACT METRIC SPACES
}

\author{
PHILIP L. BOWERS
}

\begin{abstract}
It is proved that a connected complete separable ANR $Z$ that satisfies the discrete $n$-cells property admits dense embeddings of every $n$-dimensional $\sigma$-compact, nowhere locally compact metric space $X(n \in N \cup\{0, \infty\})$. More generally, the collection of dense embeddings forms a dense $G_{\delta}$-subset of the collection of dense maps of $X$ into $Z$. In particular, the collection of dense embeddings of an arbitrary $\sigma$-compact, nowhere locally compact metric space into Hilbert space forms such a dense $G_{\delta}$-subset. This generalizes and extends a result of Curtis $\left[\mathbf{C u}_{1}\right]$.
\end{abstract}

0. Introduction. In $\left[\mathrm{Cu}_{1}\right], \mathrm{D}$. W. Curtis constructs dense embeddings of $\sigma$-compact, nowhere locally compact metric spaces into the separable Hilbert space $l_{2}$. In this paper, we extend and generalize this result in two distinct ways: first, we show that any dense map of a $\sigma$-compact, nowhere locally compact space into Hilbert space is strongly approximable by dense embeddings, and second, we extend this result to finite-dimensional analogs of Hilbert space. Specifically, we prove

THEOREM. Let $Z$ be a complete separable ANR that satisfies the discrete $n$-cells property for some $n \in N \cup\{0, \infty\}$ and let $X$ be a $\sigma$-compact, nowhere locally compact metric space of dimension at most $n$. Then for every map $f: X \rightarrow Z$ such that $f(X)$ is dense in $Z$ and for every open cover $\mathscr{U}$ of $Z$, there exists an embedding $g: X \rightarrow Z$ such that $g(X)$ is dense in $Z$ and $g$ is $\mathscr{U}$-close to $f$.

We actually prove a slightly stronger version of the Theorem. We prove that the collection of dense embeddings of $X$ into $Z$ forms a dense $G_{\delta}$-subset of the collection of dense maps of $X$ into $Z$ topologized by the limitation topology.

COROLlary. Let $n$ and $Z$ be as in the Theorem with the added assumption that $Z$ is connected. Then $Z$ admits a dense embedding of every $\sigma$-compact, nowhere locally compact metric space $X$ of dimension at most $n$.

Proof. It suffices to show that there is at least one map $X \rightarrow Z$ whose image is dense in $Z$. Let $R$ denote the following subset of the plane:

$$
R=([1, \infty) \times\{0\}) \cup(N \times[0, \infty)) .
$$

$R$ is a separable AR and since $X$ is noncompact, there is a map of $X$ onto a dense subset of $R$. (Let $\left\{x_{i}\right\}$ be a countable closed discrete subset of $X$ and $\left\{r_{i}\right\}$ a

Received by the editors October 8, 1984 and, in revised form, November 26, 1984.

1980 Mathematics Subject Classification. Primary 54C25; Secondary 54D45, 54F45.

Key words and phrases. Discrete $n$-cells property, sigma-compact space, nowhere locally compact space, dense embedding, limitation topology. 
countable dense subset of $R$ and extend $f:\left\{x_{i}\right\} \rightarrow\left\{r_{i}\right\}$ defined by $f\left(x_{i}\right)=r_{i}$ to all of $X$.) Hence, it suffices to show that there is a map of $R$ onto a dense subset of $Z$. Let $\mathscr{U}=\left\{U_{n}\right\}_{n=1}^{\infty}$ be a countable open cover of $Z$ such that $\mathscr{U}$-close maps are homotopic. Since $Z$ is a connected ANR, hence path-connected, there is a map $g$ : $R \rightarrow Z$ such that $g(\{n\} \times[0, \infty))=g((n, 0)) \in U_{n}$. For each $n \in N$, let $\{z(n, i)\}_{i=1}^{\infty}$ be a countable dense subset of $U_{n}$ and define $h$ on $R^{\prime}=([1, \infty) \times\{0\}) \cup(N \times N)$ by $h=g$ on $[1, \infty) \times\{0\}$ and $h((n, i))=z(n, i)$. Then $h$ is continuous and $\mathscr{U}$-close to $g \mid R^{\prime}$, hence $h$ is homotopic to $g \mid R^{\prime}$. Since $g \mid R^{\prime}$ extends to a map defined on $R$, the Homotopy Extension Theorem guarantees that $h$ extends to a map defined on $R$ that necessarily has dense image in $Z$.

Curtis' result is recovered from the Corollary by recalling that the separable Hilbert space $l_{2}$ is a complete AR that satisfies the discrete approximation property ( = discrete $\infty$-cells property).

Our basic approach to the proof of the Theorem is to use the function space techniques employed by $\mathrm{H}$. Torunczyk in $\left[\mathbf{T o}_{1}\right]$ and especially $\left[\mathbf{T o}_{\mathbf{2}}\right]$ (the primary reference for these techniques is $\S 1$ of $\left[\mathrm{To}_{2}\right]$ ). $\S 1$ of this paper introduces definitions and basic concepts. In particular, we define the discrete cells properties and state the function space results that we need for the proof of the Theorem. $\$ 2$ contains a proof of the Theorem and $\S 3$ discusses some open questions.

In the proof of the Theorem in $\$ 2$, we use the fact that if $Z$ is a complete separable ANR that satisfies the discrete $n$-cells property, then any map of an $n$-dimensional space $X$ into $Z$ is arbitrarily close to an embedding, with closeness measured by open covers of $Z$. This result and its proof are very similar to certain results and their corresponding proofs that Torunczyk presents in [ $\left.\mathbf{T o}_{2}\right]$ (see Lemmas 3.6-3.8 of $\left[\mathbf{T o}_{2}\right]$ ); however, the results of $\left[\mathbf{T o}_{2}\right]$ do not quite provide a proof for this result. Presumably, this result is known to Torunczyk and to others in this field, but the author could not find them in the literature. For this reason, we include an Appendix that contains a proof of a slightly more general version of this result.

Terminology and notation. All spaces are assumed to be separable and metrizable and maps are continuous functions. If $K$ is an abstract simplicial complex, then $K^{(n)}$ denotes its $n$-skeleton and $|K|$ its standard geometric realization equipped with the metric topology [Hu]. $N$ denotes the collection of positive integers.

1. Preliminaries. A collection $\mathscr{D}$ of subsets of a space $Z$ is discrete in $Z$ provided every point in $Z$ has a neighborhood that meets at most one member of $\mathscr{D}$. A space $Z$ satisfies the discrete $n$-cells property for some $n \in N \cup\{0, \infty\}$ if, for each map $f$ : $\oplus_{i=1}^{\infty} I_{i}^{n} \rightarrow Z$ of the countable free union of $n$-cells into $Z(\infty$-cell $=$ Hilbert cube) and each open cover $\mathscr{U}$ of $Z$, there exists a map $g: \oplus_{i=1}^{\infty} I_{i}^{n} \rightarrow Z$ such that $f$ and $g$ are $\mathscr{U}$-close and $\left\{g\left(I_{i}^{n}\right)\right\}_{i=1}^{\infty}$ is discrete in $Z$. The separable Hilbert space $l_{2}$ satisfies the discrete $\infty$-cells property, usually referred to as the discrete approximation property [To $\mathbf{T o}_{2}$ ], and the author has constructed for each $n \in N \cup\{0\}$, examples of complete $(n+1)$-dimensional AR's that satisfy the discrete $n$-cells property $\left[\mathbf{B o}_{1}\right]$. For further results about discrete cells properties, see $\left[\mathbf{T o}_{2}, \mathbf{A C M}, \mathbf{C u}_{2}, \mathbf{B o}_{1}, \mathbf{B o}_{2}\right]$.

For spaces $X$ and $Z, C(X, Z)$ denotes the set of all maps from $X$ to $Z$ equipped with the limitation topology $\left[\mathbf{T o}_{2}\right]$ in which each $f \in C(X, Z)$ has $\{B(f, \mathscr{U}) \mid \mathscr{U}$ is an 
open cover of $Z\}$ as a basis of open neighborhoods where $B(f, \mathscr{U})=\{g \in$ $C(X, Z) \mid g$ is $\mathscr{U}$-close to $f\}$. If $Z$ is metrizable, the limitation topology on $C(X, Z)$ coincides with the topology of uniform convergence with respect to all metrics for $Z$. $D(X, Z)$ denotes the subspace of $C(X, Z)$ that consists of all maps $f$ in $C(X, Z)$ with dense image in $Z$. $\operatorname{Emb}(X, Z)$ denotes the subspace of $C(X, Z)$ that consists of all embeddings. The following lemma is proved in the Appendix at the end of this paper (see discussion in the Introduction).

LEMMA 1.1. If $X$ is a separable metric space of dimension at most $n$ and $Z$ is a complete separable ANR that satisfies the discrete $n$-cells property for some $n \in N \cup$ $\{0, \infty\}$, then $\operatorname{Emb}(X, Z)$ is dense in $C(X, Z)$.

If $F \subset C(X, Z)$ and $d$ is a metric for $Z$, then $F_{d}$ denotes the $d$-closure of $F$; that is, $f \in F_{d}$ if and only if $f$ is the $d$-uniform limit of maps from $F$.

LEMMA 1.2 (SEE $\left.\left[\mathrm{To}_{2}\right]\right)$. Let $Z$ be completely metrizable, $F$ a subspace of $C(X, Z)$, and $U_{n}$ for $n \in N$ open subsets of $C(X, Z)$. If $U_{n} \cap F$ is dense in $F$ for each $n$, then maps in $F$ are approximable by elements (that is, are in the closure) of $\cap_{n=1}^{\infty} U_{n} \cap F_{d}$, where $d$ is any metric for $Z$. In particular, $C(X, Z)$ is a Baire space.

LEMMA 1.3. If $d$ is a metric on $Z$, then $D(X, Z)_{d}=D(X, Z)$.

The proofs of Lemmas 1.2 and 1.3 are omitted.

2. Dense embeddings of $\sigma$-compact, nowhere locally compact spaces. Throughout this section, $X$ denotes a $\sigma$-compact, nowhere locally compact metric space and $d$ denotes a fixed bounded metric on the space $Z$. As in $\left[\mathbf{C u}_{1}\right]$, since $X$ is $\sigma$-compact, there exists a countable collection $P=\left\{\left(K_{n}, C_{n}\right)\right\}_{n=1}^{\infty}$ of pairs of nonempty, disjoint closed subsets of $X$ such that

(i) each $K_{n}$ is compact,

(ii) for any neighborhood $U$ of a point $x$, there is an $n \in N$ such that $x \in K_{n} \subset X$ $-C_{n} \subset U$,

(iii) for any pair $x, y$ of distinct points in $X$, there is an $n \in N$ such that $x \in K_{n}$ and $y \in C_{n}$.

(Construction $\left[\mathbf{C u}_{1}\right]$. Let $\left\{B_{i}\right\}$ be a countable open base for $X$ and write $X=$ $\bigcup_{i=1}^{\infty} X_{i}$, each $X_{i}$ compact. Obtain $P$ by considering pairs $\left(X_{i} \cap \mathrm{Cl}_{X} B_{j}, X-B_{k}\right)$, where $\mathrm{Cl}_{X} B_{j} \subset B_{k}$.)

For $n \in N$, define $U_{n}$ by

$$
\begin{aligned}
U_{n} & =\left\{f \in C(X, Z) \mid d\left(f\left(K_{n}\right), f\left(C_{n}\right)\right)>0\right\} \\
& =\left\{f \in C(X, Z) \mid f\left(K_{n}\right) \cap \mathrm{Cl}_{Z}\left(f\left(C_{n}\right)\right)=\varnothing\right\} .
\end{aligned}
$$

LEMMA 2.1. Each $U_{n}$ is open in $C(X, Z)$ and $\operatorname{Emb}(X, Z)=\bigcap_{n=1}^{\infty} U_{n}$.

The proof of Lemma 2.1 is left to the reader. Use (ii) to show that $f^{-1}: f(X) \rightarrow X$ is continuous for each $f \in \bigcap_{n=1}^{\infty} U_{n}$. See $\left[\mathbf{C u}_{1}\right]$. 
THEOREM 2.2. If $X$ has dimension at most $n$ and $Z$ is a complete separable ANR that satisfies the discrete $n$-cells property for some $n \in N \cup\{0, \infty\}$, then for every $k \in N$, $U_{k} \cap D(X, Z)$ is dense in $D(X, Z)$.

Combining Lemmas 1.2, 1.3, and 2.1 with Theorem 2.2 provides the following

Corollary 2.3. Given the hypotheses of Theorem $2.2, \operatorname{Emb}(X, Z) \cap D(X, Z)$ is a dense $G_{\delta}$-subset of $D(X, Z)$.

The Theorem stated in the Introduction is a direct consequence of Corollary 2.3.

Proof of Theorem 2.2. Fix $k \in N, f \in D(X, Z)$, an arbitrary open cover $\mathscr{U}$ of $Z$, and a locally finite refinement $\mathscr{V}$ of $\mathscr{U}$ such that $\mathrm{st}^{2} \mathscr{V}$-close maps into $Z$ are $\mathscr{U}$-homotopic. Let $W$ and $\tilde{W}$ be open subsets of $Z$ such that

$$
f\left(K_{k}\right) \subset \tilde{W} \subset \mathrm{Cl}_{Z} \tilde{W} \subset W \subset \operatorname{st}\left(f\left(K_{k}\right), \mathscr{V}\right)
$$

and choose open refinements $\mathscr{A}<\mathscr{B}<\mathscr{V}$ such that $\mathscr{A}$-close maps in $Z$ are $\mathscr{B}$-homotopic and

$$
\operatorname{st}\left(f\left(K_{k}\right), \mathscr{B}\right) \cap \operatorname{st}(Z-\tilde{W}, \mathscr{B})=\varnothing .
$$

According to Lemma 1.1, we can choose an embedding $g: f^{-1}\left(\mathrm{Cl}_{Z} \tilde{W}\right) \rightarrow Z$ that is $\mathscr{A}$-close and therefore $\mathscr{B}$-homotopic to $f \mid f^{-1}\left(\mathrm{Cl}_{Z} \tilde{W}\right)$. Extend $g$ on $f^{-1}(Z-W)$ by letting $g=f$ on $f^{-1}(Z-W)$ and use the Homotopy Extension Theorem [Hu] to further extend $g$ to a map $g: X \rightarrow Z$ that is $\mathscr{B}$-close to $f$.

We claim that $g \in U_{k}$, that is, we claim that $g\left(K_{k}\right) \cap\left[\mathrm{Cl}_{Z} g\left(C_{k}\right)\right]=\varnothing$. Indeed, suppose that $\left\{c_{i}\right\} \subset C_{k}$ is a sequence and $g\left(c_{i}\right) \rightarrow z$ for some $z \in Z$. If infinitely many $f\left(c_{i}\right)$ belong to $Z-\tilde{W}$, then for infinitely many $i, g\left(c_{i}\right) \in \operatorname{st}(Z-\tilde{W}, \mathscr{B})$ and (*) implies that $z \notin \operatorname{st}\left(f\left(K_{k}\right), \mathscr{B}\right)$. But $g\left(K_{k}\right) \subset \operatorname{st}\left(f\left(K_{k}\right), \mathscr{B}\right)$, so $z \notin g\left(K_{k}\right)$. Assume then that $f\left(c_{i}\right) \in \tilde{W}$ for all $i$ and suppose that $z=g(x)$ for some $x \in K_{k}$. Then $g\left(c_{i}\right) \rightarrow g(x)$ and since $\left\{c_{i}\right\} \cup\{x\} \subset f^{-1}(\tilde{W})$ and $g$ is an embedding on $f^{-1}(\tilde{W}), c_{i} \rightarrow x$. This contradicts the fact that $C_{k}$ is closed in $X$ and $K_{k} \cap C_{k}=\varnothing$.

We have yet to invoke the nowhere local compactness of $X$. We wish to adjust $g$ to obtain a map $h$ close to $g$ that remains in $U_{k}$ and has dense image in $Z$. Since $g=f$ on $f^{-1}(Z-W)$ and $f$ has dense image in $Z, Z-W \subset \mathrm{Cl}_{Z} g(X)$. Since $\mathscr{V}$ is locally finite and $f\left(K_{k}\right)$ is compact, $\operatorname{st}\left(f\left(K_{k}\right), \mathscr{V}\right)=V_{1} \cup V_{2} \cup \cdots \cup V_{r}$ for some $V_{i} \in \mathscr{V}$, $i=1, \ldots, r$, some $r \in N$. For each $i=1, \ldots, r$, choose $B_{i} \in \mathscr{B}$ such that $B_{i} \cap V_{i} \cap$ $f\left(K_{k}\right) \neq \varnothing$. Then $g^{-1}\left(\operatorname{st}\left(B_{i}, \mathscr{B}\right)\right) \cap K_{k} \neq \varnothing$ and since $C_{k}$ is closed, $K_{k}$ is compact, and $X$ is nowhere locally compact, $g^{-1}\left(\operatorname{st}\left(B_{i}, \mathscr{B}\right)\right)-\left(K_{k} \cup C_{k}\right)$ is a nonempty open subset of $X$. Thus, for each $i=1, \ldots, r$, we may choose a closed discrete countable subset $D_{i}=\{x(i, j)\}_{j=1}^{\infty}$ of $X$ such that $D_{i} \subset g^{-1}\left(\operatorname{st}\left(B_{i}, \mathscr{B}\right)\right)-\left(K_{k} \cup C_{k}\right)$ and we may assume that $\left\{D_{i}\right\}_{i=1}^{r}$ is a pairwise disjoint collection. Since $\operatorname{st}\left(B_{i}, \mathscr{B}\right) \cap(Z-$ $W)=\varnothing$ (by $(*))$ and $f^{-1}(Z-W) \subset g^{-1}(Z-W), D_{i} \cap f^{-1}(Z-W)=\varnothing$ for each $i=1, \ldots, r$. For each $i=1, \ldots, r$, let $\{z(i, j)\}_{i=1}^{\infty}$ be a countable dense subset of $V_{i}$ and define a map $h: D \rightarrow Z$ where $D=f^{-1}(Z-W) \cup K_{k} \cup C_{k} \cup D_{1} \cup$ $\cdots \cup D_{r}$ by $h=g$ on $f^{-1}(Z-W) \cup K_{k} \cup C_{k}$ and $h(x(i, j))=z(i, j)$ for all $x(i, j) \in D_{1} \cup \cdots \cup D_{r}$. Then $h$ is well defined and continuous and $\mathrm{st}^{2} \mathscr{V}$-close to $g \mid D$ and therefore, $h$ is $\mathscr{U}$-homotopic to $g \mid D$. Since $g$ extends to $X$, the Homotopy 
Extension Theorem implies that $h$ extends to a map $h: X \rightarrow Z$ that is $\mathscr{U}$-close to $g$. Since $h\left(K_{k}\right)=g\left(K_{k}\right)$ and $h\left(C_{k}\right)=g\left(C_{k}\right), h \in U_{k}$. Since $h\left(D_{i}\right)$ is dense in $V_{i}$ and $W \subset \bigcup_{i=1}^{r} V_{i}, h$ has dense image in $Z$. Therefore, we have found a map $h$ in $U_{k} \cap D(X, Z)$ that is st $\mathscr{U}$-close to $f$ and this implies that $U_{k} \cap D(X, Z)$ is dense in $D(X, Z)$.

3. Final remarks. The obvious necessary conditions for a metric space to be densely embeddable in Hilbert space are separability and nowhere local compactness and it remains an open question whether these conditions are sufficient $\left[\mathbf{C u}_{1}\right]$.

Question A. If $X$ is a nowhere locally compact separable metric space, does $X$ admit a dense embedding into $l_{2}$ ?

Question B. If $X$ is a nowhere locally compact separable metric space of dimension at most $n$ and $Z$ is a connected complete separable ANR that satisfies the discrete $n$-cells property for some $n \in N \cup\{0, \infty\}$, does $X$ admit a dense embedding into $Z$ ?

Ad hoc methods provide an affirmative answer to Question B whenever $n=0$. Indeed, if $\tilde{X}$ is a 0 -dimensional compactification of $X$ [HW], then $\tilde{X}-X$ is dense in $\tilde{X}$ and $X \subset \tilde{X}-D$ for some countable dense subset $D$ of $\tilde{X}$. The classical Alexandroff-Urysohn characterization of the irrationals $P[\mathbf{A U}]$ as the unique 0 -dimensional complete, nowhere locally compact space applies to show that $\tilde{X}-D$ is homeomorphic to $P$. Thus, it suffices to show that $P$ admits a dense embedding into $Z$. Let $E \subset P$ and $F \subset Z$ be countable dense subsets and let $h: E \rightarrow F$ be a homeomorphism. Then $h$ extends to a homeomorphism $h_{*}$ of $E_{*}$ onto $F_{*}$ where $E_{*}$ and $F_{*}$ are $G_{\delta}$-subsets of $P$ and $Z$, respectively [La, Wi, Theorem 24.9]. The Alexandroff-Urysohn characterization applies then to the topologically complete nowhere locally compact space $E_{*}$. Thus $h_{*}$ is an embedding of $E_{*} \approx P$ into $Z$ and, since $h_{*}\left(E_{*}\right) \supset F, h_{*}$ is a dense embedding.

Notice that we never use the discrete 0 -cells property nor the fact that $Z$ is an ANR in the discussion above. Hence, every complete separable space with no isolated points admits dense embeddings of nowhere locally compact, separable metric spaces of dimension zero.

Appendix. Dense $G_{\delta}$-subsets of $C(X, Z)$. Recall that $C(X, Z)$ denotes the space of maps $X \rightarrow Z$ equipped with the limitation topology and $\operatorname{Emb}(X, Z)$ is the subspace of $C(X, Z)$ that consists of all embeddings of $X$ into $Z$. Denote the subspace of $C(X, Z)$ that consists of all closed embeddings by $\overline{\operatorname{Emb}}(X, Z)$.

THEOREM. Let $X$ be a separable metric space of dimension at most $n$ and let $Z$ be a complete separable ANR that satisfies the discrete n-cells property for some $n \in N \cup$ $\{0, \infty\}$. Then

(1) $\operatorname{Emb}(X, Z)$ contains a dense $G_{\delta}$-subset of $C(X, Z)$,

(2) if $X$ is complete, $\overline{\operatorname{Emb}}(X, Z)$ is a dense $G_{\delta}$-subset of $C(X, Z)$,

(3) if $X$ is $\sigma$-compact, $\operatorname{Emb}(X, Z)$ is a dense $G_{\delta}$-subset of $C(X, Z)$.

For the proof of the Theorem we need some preliminary definitions and lemmas. Given a cover $\mathscr{U}$ of $X$, a map $f: X \rightarrow Z$ is a $\mathscr{U}$-map if there is a cover $\mathscr{V}$ of $Z$ with $f^{-1}(\mathscr{V})$ refining $\mathscr{U}$. 


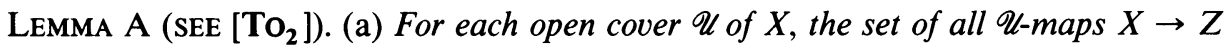
is open in $C(X, Z)$.

(b) Let $d$ be any metric for $X$ and, for each $n \in N$, let $\mathscr{U}_{n}$ be an open cover of $X$ such that $\operatorname{diam}_{d} U<1 / n$ for all $U \in \mathscr{U}_{n}$. Let $\mathscr{F}_{n}=\left\{f \in C(X, Z) \mid f\right.$ is a $\mathscr{U}_{n}$-map $\}$. Then $\bigcap_{n=1}^{\infty} \mathscr{F}_{n} \subset \operatorname{Emb}(X, Z)$.

(c) Let $d$ be a complete metric on $X$ and define $\mathscr{U}_{n}$ and $\mathscr{F}_{n}$ as in (b). Then $\bigcap_{n=1}^{\infty} \mathscr{F}_{n}$ $=\overline{\mathrm{Emb}}(X, Z)$.

The proof of Lemma A is omitted. Parts (a) and (c) appear in $\$ 1$ of $\left[\mathbf{T o}_{2}\right]$ and the proof of part (b) is straightforward.

LEMMA B. Let $X$ be a separable n-dimensional $(n \in N)$ metric space and $\mathscr{U}$ an arbitrary open cover of $X$. Then there is a countable open star-finite refinement $\mathscr{V}$ of $\mathscr{U}$ of order less than or equal to $n$.

It is well known that there are star-finite refinements of $\mathscr{U}$ and that there are refinements of $\mathscr{U}$ of order less than or equal to $n$ [Pe]; however, the author could find no reference for the fact that one can obtain a refinement of $\mathscr{U}$ that is simultaneously star-finite and of order not exceeding $n$. Therefore, we include a proof of Lemma B.

Proof of Lemma B. First, embed $X$ in a compact $n$-dimensional space $\bar{X}$ [HW] and let $\overline{\mathscr{U}}$ denote a collection of open subsets of $\bar{X}$ such that $\mathscr{U}=\{\bar{U} \cap X \mid \bar{U} \in \overline{\mathscr{U}}\}$. Let $Y=\bigcup\{\bar{U} \mid \bar{U} \in \overline{\mathscr{U}}\}$, an open subset of $\bar{X}$, and write $Y=\bigcup_{i=1}^{\infty} Y_{i}$ where $Y_{1}=\varnothing$, each $Y_{i}$ is compact, and $Y_{i} \subset \stackrel{\circ}{Y}_{i+1}$ where $\stackrel{\circ}{Y}_{i}=\operatorname{Int}_{Y} Y_{i}$ for each $i \in N$. Let $\mathscr{A}$ be an open refinement of $\overline{\mathscr{U}}$ such that if $A \in \mathscr{A}$ and $A \cap Y_{i} \neq \varnothing$, then $A \subset Y_{i+1}$. This implies that if $A \cap\left(Y_{i+1}-Y_{i}\right) \neq \varnothing$, then $A \cap Y_{i-1}=\varnothing$. Since $Y$ is $n$-dimensional, there is an open refinement $\mathscr{B}$ of $\mathscr{A}$ of order less than or equal to $n$ [Pe, Theorem 4.3]. For each $i \in N$, let $\mathscr{B}_{i}$ be a minimal finite subcollection of $\mathscr{B}$ that covers $Y_{i+1}-\dot{Y}_{i}$ and let $\overline{\mathscr{V}}=\bigcup_{i=1}^{\infty} \mathscr{B}_{i}$. Clearly $\overline{\mathscr{V}}$ is an open cover of $Y$ refining $\overline{\mathscr{U}}$; thus, $\mathscr{V}=\{\bar{V}$ $\cap X \mid \bar{V} \in \bar{V}\}$ is an open refinement of $\mathscr{U} . \mathscr{V}$ has order less than or equal to $n$ since $\mathscr{B}$ (and therefore $\overline{\mathscr{V}}$ ) does and the reader may use the special properties of $\mathscr{A}$ to show that $\mathscr{V}$ is star-finite.

The following lemma and its proof are very similar to Lemma 3.6 of $\left[\mathbf{T o}_{2}\right]$ and its proof.

LeMma C. Let $K$ be a locally finite $n$-dimensional $(n \in N)$ complex with at most countably many vertices and let $Z$ be a complete separable ANR that satisfies the discrete $n$-cells property. Let $\mathscr{U}$ be the open cover of $|K|$ by open stars of vertices of $K$. Then the collection of $\mathscr{U}$-maps $|K| \rightarrow Z$ is dense in $C(|K|, Z)$ and $\overline{\operatorname{Emb}}(|K|, Z)$ is a dense $G_{\delta}$-subset of $C(|K|, Z)$.

Proof. If $\operatorname{dim} K=0$, then the result follows from the discrete 0 -cells property for $Z$. Suppose that $\operatorname{dim} K=n$ and the lemma is proved for complexes of dimension $n-1$. Let $f:|K| \rightarrow Z$ be a map and let $\mathscr{W}$ be an open cover of $Z$. Since $Z$ is an ANR, the inductive assumption allows us to assume that $f|| K^{(n-1)} \mid$ is a $\mathscr{U}$-map. Let $P$ be a closed neighborhood of $\left|K^{(n-1)}\right|$ in $|K|$ with $f \mid P$ a $\mathscr{U}$-map $\left[\mathbf{T o}_{2}, 1.4(\mathrm{c})\right]$.

Choose a cover $\overline{\mathscr{W}}$ of $Z$ refining $\mathscr{W}$ such that if $g:|K| \rightarrow Z$ is $\overline{\mathscr{W}}$-close to $f$, then $g \mid P$ is a $\mathscr{U}$-map. Let $\left\{C_{\sigma} \mid \sigma \in K^{(n)}\right\}$ be a cover of $|K|-P$, discrete in $|K|$, such that 
$C_{\sigma} \approx I^{n}$ and $C_{\sigma} \subset$ Int $\sigma$ for each $\sigma \in K^{(n)}\left[\mathbf{T o}_{2}, 3.5\right]$. Since $Z$ is an ANR and satisfies the discrete $n$-cells property, there is a map $g:|K| \rightarrow Z$ that is $\overline{\mathscr{W}}$-close to $f$ for which $\left\{g\left(C_{0}\right) \mid \sigma \in K^{(n)}\right\}$ is discrete. Since $Z$ satisfies the discrete $n$-cells property, every compact subset of $Z$ is a $Z_{n}$-set in $Z$; thus, since $g(P)$ is $\sigma$-compact, $g(P)$ is a $\sigma-Z_{n}$-set in the complete ANR $Z$. Therefore, we may assume that for each $\sigma \in K^{(n)}$,

$$
g\left(C_{\sigma}\right) \cap g(P-\text { Int } \sigma)=\varnothing .
$$

Our claim is that $g$ is a $\mathscr{U}$-map. Since $g$ is $\overline{\mathscr{W}}$-close to $f, g \mid P$ is a $\mathscr{U}$-map. Since $\mathscr{U}$ is a cover of $|K|$ by relatively compact sets (recall that $K$ is locally finite) and $g \mid P$ is a $\mathscr{U}$-map, $g \mid P$ is a closed map (in fact, proper). Therefore, $g(P-$ Int $\sigma$ ) is closed in $Z$ for all $\sigma \in K^{(n)}$. If $z \in g\left(C_{\sigma}\right)$ for some $\sigma \in K^{(n)}$, let $A_{z}^{\prime}$ be a neighborhood of $z$ such that $A_{z}^{\prime}$ misses $g\left(C_{\tau}\right)$ for every $\tau \in K^{(n)}-\{\sigma\}$ and let $A_{z}=A_{z}^{\prime}-g(P-$ Int $\sigma)$. Then $A_{z}$ is a neighborhood of $z$ since (*) holds and $g(P-$ Int $\sigma)$ is closed. We have $g^{-1}\left(A_{z}\right) \subset$ Int $\sigma \subset \operatorname{St}(v) \in \mathscr{U}$ for $v \in \sigma^{(0)}$. If $z \in Z-\cup\left\{g\left(C_{\sigma}\right) \mid \sigma \in K^{(n)}\right\}$ let $A_{z}$ be a neighborhood of $z$ such that $A_{z}$ misses $g\left(C_{\sigma}\right)$ for all $\sigma \in K^{(n)}$ and $(g \mid P)^{-1}\left(A_{z}\right) \subset U$ for some $U \in \mathscr{U}$. Then $g^{-1}(\mathscr{A})$ refines $\mathscr{U}$ for $\mathscr{A}=\left\{A_{z} \mid z \in Z\right\}$. Therefore $g$ is a $\mathscr{U}$-map and the collection of $\mathscr{U}$-maps $|K| \rightarrow Z$ is dense in $C(|K|, Z)$.

Now let $d$ be a complete metric on $|K|$ and, for each $n \in N$, choose a triangulation $K_{n}$ of $K$ such that the open stars of vertices of $K_{n}$ have $d$-diameter less than $1 / n$ and let $\mathscr{U}_{n}$ denote the open cover of $|K|$ by open stars (in $K_{n}$ ) of vertices of $K_{n}$. Then $\mathscr{F}_{n}=\left\{f \in C(|K|, Z) \mid f\right.$ is a $\mathscr{U}_{n}$-map $\}$ is open and dense in $C(|K|, Z)$ and $\bigcap_{n=1}^{\infty} \mathscr{F}_{n}$ $=\overline{\mathrm{Emb}}(|K|, Z)$ is a dense $G_{\delta}$-subset of $C(|K|, Z)$ (apply Lemma A, the first part of Lemma $C$, and Lemma 1.2).

Proof of Theorem. (1) If $n=\infty$, then $Z$ is homeomorphic to $l_{2}\left[\mathbf{T o}_{2}\right]$ and the Theorem follows from the techniques of $\left[\mathbf{T o}_{2}\right]$. We assume that $n<\infty$. Let $d$ be a metric for $X$ and define $\mathscr{U}_{n}$ and $\mathscr{F}_{n}$ for $n \in N$ as in Lemma A(b). By Lemma 1.2 and Lemma $\mathrm{A}(\mathrm{b})$, it suffices to prove that $\mathscr{F}_{n}$ is dense in $C(X, Z)$ for all $n \in N$.

Let $f \in C(X, Z)$ and $\mathscr{U}$ an open cover of $Z$ and let $\tilde{\mathscr{U}}$ be a refinement of $\mathscr{U}$ such that every partial $\tilde{\mathscr{U}}$-realization of any complex extends to a full $\mathscr{U}$-realization. Use Lemma $\mathrm{B}$ to find a countable star-finite refinement $\mathscr{V}$ of a star-refinement of a common refinement of $f^{-1}(\tilde{\mathscr{U}})$ and $\mathscr{U}_{n}$ such that the order of $\mathscr{V}$ does not exceed $n$. Then $K=\mathscr{N}(\mathscr{V})$, the nerve of $\mathscr{V}$, is a countable locally finite $n$-dimensional complex and there is a canonical map $\psi: X \rightarrow|K|$ such that

$$
\left\{\psi^{-1}(\operatorname{St}(v)) \mid v \in K^{(0)}\right\}<\mathscr{V}
$$

where $\operatorname{St}(v)$ denotes the open star of $v$ in $K$. A standard argument provides a map $\phi$ : $|K| \rightarrow Z$ such that $\phi \circ \psi$ is st $\mathscr{U}$-close to $f$. Lemma C provides a closed embedding $\bar{\phi}:|K| \rightarrow Z$ such that $\bar{\phi}$ is $\mathscr{U}$-close to $\phi$ so that $g=\bar{\phi} \circ \psi$ is $\operatorname{st}^{2} \mathscr{U}$-close to $f$. The reader may check that $g \in \mathscr{F}_{n}$ so that $\mathscr{F}_{n}$ is dense in $C(X, Z)$.

(2) If $d$ is a complete metric for $X$, use Lemma $\mathrm{A}(\mathrm{c})$ in place of Lemma $\mathrm{A}(\mathrm{b})$ in the proof of (1) to prove (2).

(3) Part (1) implies that $\operatorname{Emb}(X, Z)$ is dense in $C(X, Z)$ and Lemma 2.1 implies that $\operatorname{Emb}(X, Z)$ is a $G_{\delta}$-subset of $C(X, Z)$. 


\section{REFERENCES}

[ACM] R. D. Anderson, D. W. Curtis and J. van Mill, A fake topological Hilbert space, Trans. Amer. Math. Soc. 272 (1982), 311-321.

[AU] P. Alexandroff and P. Urysohn, Über nulldimensionale Punktmengen, Math. Ann. 98 (1928), 89-106.

[Bo $\left.\mathbf{B}_{1}\right]$ P. L. Bowers, General position properties satisfied by finite products of dendrites, Trans. Amer. Math. Soc. 288 (1985), 739-753.

$\left[\mathbf{B o}_{2}\right] \ldots$ Discrete cells properties in the boundary set setting, Proc. Amer. Math. Soc. 93 (1985), $735-740$

$\left[\mathbf{C u}_{1}\right]$ D. W. Curtis, Sigma-compact, nowhere locally compact metric spaces can be densely imbedded in Hilbert sace, Topology Appl. 16 (1983), 253-257.

$\left[\mathrm{Cu}_{2}\right] \ldots$, Boundary sets in the Hilbert cube, Topology Appl. (to appear).

[Hu] S. T. Hu, Theory of retracts, Wayne State Univ. Press, Detroit, Mich., 1965.

[HW] W. Hurewicz and H. Wallman, Dimension theory, Princeton Univ. Press, Princeton, N. J., 1969.

[La] M. Lavrentieff, Contribution à la théorie des ensembles homéomorphs, Fund. Math. 6 (1924), 149-160.

[Pe] A. R. Pears, Dimension theory of general spaces, Cambridge Univ. Press, Cambridge, 1975.

$\left[\mathrm{To}_{1}\right] \mathrm{H}$. Torunczyk, On CE-images of the Hilbert cube and characterization of $Q$-manifolds, Fund. Math. 106 (1980), 31-40.

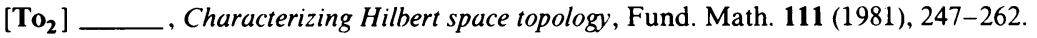

[Wi] S. Willard, General topology, Addison-Wesley, Reading, Mass., 1970.

Department of Mathematics, Florida State University, Tallahassee, Florida 32306 